\title{
Variabilidade espacial de atributos físicos de um Latossolo Vermelho após cultivo de soja
}

\author{
Diego dos Santosi ${ }^{1}$, Eduardo G. de Souza ${ }^{2}$, Lúcia H. P. Nóbrega ${ }^{2}$, \\ Claudio L. Bazzi ${ }^{3}$ \& Affonso C. Gonçalves Júnior ${ }^{4}$
}

\begin{abstract}
RESUMO
Os atributos químicos e físicos do solo apresentam, frequentemente, dependência espacial. Portanto, é imprescindível estudar sua variabilidade espacial visando ao manejo mais preciso da lavoura, a redução do impacto ambiental, a otimização do uso de máquinas e o aumento da eficiência das equipes de trabalho. Assim, o objetivo deste trabalho foi avaliar a variabilidade espacial de atributos físicos de um Latossolo Vermelho distroférrico típico após o cultivo de soja. Os dados foram coletados em uma área de 2 ha cultivada sob sistema de plantio direto utilizando-se uma grade amostral de $30 \times 33 \mathrm{~m}$, em que foram avaliados os parâmetros: macroporosidade, microporosidade, porosidade total, densidade e umidade do solo, na camada de $0-0,1 \mathrm{~m}$; após as análises exploratória e geoestatística, os modelos teóricos foram ajustados aos semivariogramas para cada atributo; já a interpolação dos dados foi realizada utilizando-se a krigagem ordinária, verificando-se que todas as variáveis estudadas apresentaram dependência espacial, que variou entre moderada (macroporosidade, porosidade total e umidade do solo) e forte (microporosidade e densidade do solo), em função do coeficiente de efeito pepita.
\end{abstract}

Palavras-chave: geoestatística, porosidade do solo, umidade do solo, densidade do solo

\section{Spatial variability of physical attributes of a distroferric Red Latosol after soybean crop}

\begin{abstract}
The chemical and physical properties of a soil often show a spatial dependence. Thus, it is important to study their spatial variability, aiming to improve the crop management, to reduce the environmental impact, to optimize the machinery use, and to increase the efficiency of staff. So, this study aimed to evaluate the spatial variability of the physical properties of a distroferric Red Latosol (Typic Haplorthox) after soybean crop. The data were collected in an area of 2 ha managed under no tillage system, using a sampling grid of $30 \times 33 \mathrm{~m}$, where it was evaluated the macro, micro and the total porosity, the bulk density and the soil water content in $0-0.1 \mathrm{~m}$ layer. After the exploratory and geostatistical analyses, the theoretical models were set to the semivariograms for each attribute. The data interpolation was performed using the ordinary kriging. All the variables showed spatial dependence, which ranged from moderate (macroporosity, total porosity and soil water content) to strong (microporosity and bulk density), according to the nugget effect coefficient.
\end{abstract}

Key words: geostatistics, soil porosity, soil water content, bulk density

UFFS, CEP 85.303-820 Laranjeiras do Sul, PR. Fone (42) 3635-8650. E-mail: diegosagro@hotmail.com 2 Programa de Pós-Graduação em Eng. Agrícola/UNIOESTE, Pesquisador de Produtividade do CNPq. CEP 85819-110 Cascavel, PR. Fone: (45) $3220-3175$. E-mail: eduardo.souza@unioeste.br; lucia.nobrega@unioeste.br

3 UTFPR, CEP 85884-000 Medianeira, PR. Fone: (45) 3240-8000. E-mail: bazzi@utfpr.edu.br

${ }^{4}$ Programa de Pós-Graduação em Agronomia/UNIOESTE. Marechal Cândido Rondon, PR. Fone: (45) 3284-7924. E-mail: affonso133@hotmail.com 


\section{INTRODUÇÃO}

O estudo de atributos do solo tem demonstrado dependência espacial frequente,dos atributos químicos e físicos do solo. Atributos como densidade e porosidade total mostram quando um solo apresenta condições adequadas ao desenvolvimento e à exploração das raízes, indicando se há problemas de compactação (Ramirez-Lopez et al., 2008).

Conforme Souza et al. (2004), os atributos físicos do solo variam entre pontos relativamente próximos em áreas de mesma unidade taxonômica, muitas vezes de forma significativa. Desta forma, a pouca coerência entre as respostas encontradas com as ferramentas clássicas da estatística fez com que a geoestatística tivesse aplicação mais adequada nessa área. Para Schaffrath et al. (2008) tal desuniformidade está associada, provavelmente, ao sistema de manejo, às culturas adotadas e às características intrínsecas do solo, relacionadas com os fatores e processos de formação.

Vieira \& Klein (2007) relataram que dentre os componentes do manejo, o preparo do solo é o que mais influi em seu comportamento físico, visto que atua diretamente em sua estrutura, causando modificações na porosidade e densidade, que afetam a retenção de água e a resistência mecânica. Entretanto, as medidas corretivas, aplicadas em situações relacionadas a problemas físicos do solo, são resolvidas por meio de práticas de mecanização usualmente realizadas de forma inadequada, sem considerar a inerente dependência espacial das propriedades físicas dos solos (Ramirez-Lopez et al., 2008).

Uma vez quantificada a dependência espacial, ela pode ser utilizada para classificação e levantamento de solos em uma área, tal como na interpolação entre observações, permitindo o mapeamento das propriedades do solo dentro da área, por meio da krigagem (Gonçalves et al., 2001). Outro uso diz respeito à identificação de áreas compactadas e ao consequente manejo localizado deste problema (Ramirez-Lopez et al., 2008). A compactação do solo aumenta a resistência mecânica ao crescimento radicular, reduz a aeração e a disponibilidade de água e nutrientes, retarda a germinação das sementes e, em consequência, reduz a produtividade agrícola (Goedert et al., 2002; Kiliç et al., 2004; Megda et al., 2008).

Dado à necessidade de entender o comportamento e relação entre as propriedades físicas do solo, o objetivo deste estudo foi verificar a variabilidade espacial da macroporosidade, microporosidade, porosidade total, densidade e umidade de um Latossolo Vermelho distroférrico típico, após o cultivo de soja.

\section{MATERIAL E MÉTODOS}

Os dados experimentais foram obtidos em maio de 2008, em área pertencente ao município de Cafelândia, $\mathrm{PR}$, localizada nas coordenadas geográficas $24^{\circ} 37^{\prime} 05^{\prime \prime} \mathrm{S}$ e $53^{\circ} 19^{\prime} 18^{\prime \prime} \mathrm{O}$, com altitude média de $550 \mathrm{~m}$, clima subtropical úmido mesotérmico e precipitação média de $1850 \mathrm{~mm}$ (IAPAR, 2009). O solo foi caracterizado como Latossolo Vermelho distroférrico típico (EMBRAPA, 2006), possuindo granulometria de 92; 363 e 545 $\mathrm{g} \mathrm{kg}^{-1}$ de areia, silte e argila, respectivamente. A área de 2 ha
(133 por $151 \mathrm{~m}$ ) vinha sendo trabalhada há 10 anos, sob sistema plantio direto, com sucessão das culturas de soja, milho (verão) e trigo (inverno). No georreferenciamento da área foi usado o receptor DGPS Trimble Geo Explorer 3, com correção pósprocessada. Para a coleta dos dados foi construída uma grade de 30 por $33 \mathrm{~m}$, obtendo-se 20 pontos amostrais nos quais foram coletadas amostras indeformadas em anéis volumétricos para determinação de atributos físicos, na camada de 0-0,1 m do perfil do solo.

Para determinar a porosidade do solo (total, macro e microporosidade), as amostras indeformadas foram saturadas durante $24 \mathrm{~h}$ em bandeja com água até dois terços da altura do anel. Após o período de saturação as amostras foram colocadas em uma mesa de tensão e submetidas ao potencial equivalente a 0,006 MPa até cessar a drenagem, utilizando-se mesa de tensão (EMBRAPA, 1997). Nas mesmas amostras foram determinadas a densidade aparente do solo, por meio do método do volume conhecido e a umidade do solo obtido com a mesa de tensão (EMBRAPA, 1997).

$\mathrm{Na}$ análise exploratória dos dados foram calculadas as medidas de posição (média, mediana), de dispersão (desviopadrão) e de forma da distribuição (coeficiente de variação, coeficiente de assimetria e coeficiente de curtose). Os coeficientes de assimetria e curtose foram comparados de acordo com Jones (1969). A hipótese de normalidade dos dados foi verificada com os testes de Anderson-Darling e Kolmogorov-Smirnov, a nível de 5\% de probabilidade, através do software Minitab 14. O coeficiente de variação (CV) foi considerado baixo quando $\mathrm{CV} \leq 10 \%$ (homocedasticidade); médio quando $10 \%<\mathrm{CV} \leq 20 \%$; alto quando $20 \%<\mathrm{CV} \leq 30 \%$ e muito alto quando $\mathrm{CV}>30 \%$ (heterocedasticidade) conforme Gomes \& Garcia (2002).

A partir da análise geoestatística foram construídos os semivariogramas para verificar a dependência espacial dos dados, utilizando-se o software ArcView 9.2. Para estimar a função semivariância experimental utilizou-se o estimador de Matheron. Os modelos teóricos utilizados foram o esférico, o exponencial e o gaussiano. Obtiveram-se os semivariogramas experimentais aplicando-se os métodos de ajuste de mínimos quadrados ordinários (OLS) e se adotando o modelo isotrópico (semivariograma unidirecional) com distância de corte de 50\% da distância máxima da área em estudo (Clark, 1979).

Selecionou-se o melhor modelo por meio da validação cruzada (Faraco et al., 2008), utilizando-se o método proposto por Bazzi et al. (2009), e o índice de comparação de erros (ICE, Eq. 1) que, na seleção de j modelos, proporciona um valor tão menor quanto mais próximo de zero for ER (Erro Médio, Eq. 4) e mais perto de um for SER (Erro médio Reduzido, Eq. 5). Portanto, na escolha entre diversos modelos o melhor é o que tiver menor ICE.

$$
\mathrm{ICE}_{\mathrm{i}}=\mathrm{A}_{\mathrm{i}}+\mathrm{B}_{\mathrm{i}}
$$

em que:

$$
A_{i}=\left\{\begin{array}{cl}
\frac{\mathrm{ABS}(\overline{\mathrm{ER}})_{\mathrm{i}}}{\operatorname{MAX}(\mathrm{ABS}(\mathrm{ER}))} & , \text { quando } \operatorname{MAX}(\mathrm{ABS}(\mathrm{SME}))>0 \\
1 & , \text { quando } \operatorname{MAX}(\mathrm{ABS}(\mathrm{SME}))=0
\end{array}\right.
$$


$B_{i}=\left\{\begin{array}{cc}\frac{\mathrm{ABS}(\mathrm{SER}-1)_{\mathrm{i}}}{\operatorname{MAX}(\mathrm{ABS}(\mathrm{SER}-1))} & , \text { quando } \operatorname{MAX}(\mathrm{ABS}(\mathrm{SD} \operatorname{RME}-1))>0 \\ 1 & , \text { quando } \operatorname{MAX}(\mathrm{ABS}(\mathrm{SD} \operatorname{RME}-1))=0\end{array}\right.$

em que:

ICE - índice de comparação de erros para o modelo i

$$
\begin{aligned}
\overline{\mathrm{ER}} & =\frac{1}{\mathrm{n}} \sum_{\mathrm{i}=1}^{\mathrm{n}} \frac{\mathrm{Z}\left(\mathrm{s}_{\mathrm{i}}\right)-\hat{\mathrm{Z}}\left(\mathrm{s}_{(\mathrm{i})}\right)}{\sigma\left(\hat{\mathrm{Z}}\left(\mathrm{s}_{(\mathrm{i})}\right)\right)} \\
\mathrm{S}_{\mathrm{ER}} & =\sqrt{\frac{1}{\mathrm{n}} \sum_{\mathrm{i}=1}^{\mathrm{n}} \frac{\left|\mathrm{Z}\left(\mathrm{s}_{\mathrm{i}}\right)-\hat{\mathrm{Z}}\left(\mathrm{s}_{(\mathrm{i})}\right)\right|}{\sigma\left(\hat{\mathrm{Z}}\left(\mathrm{s}_{(\mathrm{i})}\right)\right)}}
\end{aligned}
$$

em que:

n - é o número de dados

$\mathrm{Z}\left(\mathrm{s}_{\mathrm{i}}\right)$ - valor observado no ponto si

$\hat{\mathrm{Z}}\left(\mathrm{s}_{(\mathrm{i})}\right)$ - valor predito por krigagem ordinária no ponto si, sem considerar a observação $Z_{(\text {si) }}$

$\left.\sigma\left(\mathrm{Z}_{(\mathrm{i})}\right)\right)$ - é o desvio-padrão da krigagem no ponto si, sem considerar a observação Z(si)

O grau de dependência espacial dos semivariogramas foi avaliado pelo coeficiente de efeito pepita (E\%, Eq. 1), segundo os intervalos propostos por Cambardella et al. (1994): E\% $\leq 25$ - forte dependência espacial; $25 \leq \mathrm{E} \% \leq 75$ - moderada dependência espacial e $E \% \geq 75 \%$ - fraca dependência espacial.

$$
\mathrm{E} \%=\frac{\mathrm{c}_{0}}{\mathrm{c}_{1}+\mathrm{c}_{0}} \times 100
$$

Para avaliar o grau de correlação entre as variáveis utilizou-se o coeficiente de correlação linear de Pearson (R) e o coeficiente de correlação não-paramétrica de Spearman (Rs), calculados por meio do software Minitab 14 e classificados conforme interpretação adaptada de Zou et al. (2003). O coeficiente de Spearman foi usado para investigar se os dados formam ou não uma nuvem comportada, com alguns pontos bem distantes dos demais ou se existe uma relação crescente ou decrescente num formato não linear. Os mapas temáticos foram elaborados utilizando-se os parâmetros de efeito pepita, patamar e alcance, fornecidos pelo software ArcView 9.2.

\section{RESULTADOS E DISCUSSÃO}

Os resultados referentes à estatística descritiva (Tabela 1) indicaram normalidade dos dados para todas as variáveis estudada, com distribuição simétrica e mesocúrtica para a variável porosidade total, densidade e umidade do solo. A variável macroporosidade apresentou distribuição assimétrica positiva e mesocúrtica, enquanto a microporosidade apresentou uma distribuição leptocúrtica e assimétrica negativa.

Os coeficientes de variação $(\mathrm{CV})$ indicaram homogeneidade dos dados ( $\mathrm{CV} \leq 10 \%$ ) segundo Gomes \& Garcia (2002). Como exceção, a macroporosidade teve $\mathrm{CV}=30 \%$ (alto), em concordância com os resultados obtidos por Souza et al. (2004) e Schaffrath et al. (2008) em áreas cultivadas sob sistema de plantio direto.

Constata-se que os valores de densidade do solo apresentaram valores entre 1,16 e 1,43 $\mathrm{kg} \mathrm{m}^{-3}$, semelhantes aos valores obtidos por Faraco et al. (2008). Considerando os valores encontrados por Thimóteo et al. (2001), Souza et al. (2004) e Schaffrath et al. (2008), verificou-se que a microporosidade apresentou valores altos (média de $0,46 \mathrm{~m}^{3} \mathrm{~m}^{-3}$ ) e a macroporosidade indicou valores baixos (média de $0,08 \mathrm{~m}^{3} \mathrm{~m}^{-3}$ ). Segundo Megda et al. (2008), o crescimento das raízes pode ser prejudicado quando a macroporosidade do solo é reduzida a valor inferior a $0,15 \mathrm{~m}^{3} \mathrm{~m}^{-3}$. Os resultados obtidos podem ser consequência do tráfego de máquinas e implementos agrícolas, que modificam o tamanho de agregados e aumentam a proporção de microporos em relação aos macroporos, conforme relatado por Souza et al. (2006).

A seleção dos modelos deu-se por meio do menor índice de comparação de erros (ICE, Tabela 2), obtido a partir da validação cruzada. Os dois modelos teóricos de semivariograma selecionados foram o exponencial, para as variáveis microporosidade e densidade do solo, e gaussiano para as variáveis macroporosidade, porosidade total e umidade do solo, discordando com os modelos obtidos por Schaffrath et al. (2008) e Souza et al. (2004).

Tabela 2. Índice de comparação de erros (ICE) para seleção do melhor modelo teórico

\begin{tabular}{lccc}
\hline \multirow{1}{*}{ Atributo } & \multicolumn{3}{c}{ ICE do Modelo } \\
\cline { 2 - 4 } & Esférico & Exponencial & Gaussiano \\
Macroporosidade & 1,834 & 1,915 & 1,794 \\
Microporosidade & 1,934 & 1,575 & 1,906 \\
Porosidade total & 2,000 & 1,794 & 1,517 \\
Densidade & 1,885 & 1,008 & 1,931 \\
Umidade & 1,620 & 2,000 & 1,352 \\
\hline
\end{tabular}

Tabela 1. Estatística descritiva da macroporosidade $\left(\mathrm{m}^{3} \mathrm{~m}^{-3}\right)$, microporosidade $\left(\mathrm{m}^{3} \mathrm{~m}^{-3}\right)$ porosidade total $\left(\mathrm{m}^{3} \mathrm{~m}^{-3}\right)$, densidade $\left(\mathrm{kg} \mathrm{dm}^{-3}\right)$ e umidade do solo $\left(\mathrm{m}^{3} \mathrm{~m}^{-3}\right)$

\begin{tabular}{lccccccrcr}
\hline \multicolumn{1}{c}{ Atributo } & Mínimo & Média & Mediana & Máximo & DP & CV (\%) & Assimetria & Curtose $^{\text {Normal }^{*}}$ \\
Macroporosidade & 0,05 & 0,08 & 0,07 & 0,14 & 0,023 & 30 & $1,20(\mathrm{~b})$ & $1,94(\mathrm{~A})$ & $\mathrm{sim}$ \\
Microporosidade & 0,42 & 0,46 & 0,47 & 0,49 & 0,016 & 3 & $-1,57(\mathrm{C})$ & $3,42(\mathrm{C})$ & $\mathrm{sim}$ \\
Porosidade total & 0,52 & 0,54 & 0,54 & 0,58 & 0,018 & 3 & $0,33(\mathrm{a})$ & $-0,86(\mathrm{~A})$ & $\mathrm{sim}$ \\
Densidade & 1,16 & 1,32 & 1,33 & 1,43 & 0,069 & 5 & $-0,41(\mathrm{a})$ & $0,13(\mathrm{~A})$ & $\mathrm{sim}$ \\
Umidade & 0,33 & 0,35 & 0,35 & 0,39 & 0,015 & 4 & $0,72(\mathrm{a})$ & $0,90(\mathrm{~A})$ & $\mathrm{sim}$ \\
\hline
\end{tabular}

* Teste de normalidade Anderson-Darling e Kolmogorov-Smirnov; Assimetria - distribuição simétrica (a), assimetria; positiva (b), assimetria negativa (c); Curtose - mesocúrtica (A), platicúrtica (B), leptocúrtica (C); DP - desvio padrão 
Todas as variáveis analisadas apresentaram dependência espacial (Tabela 3). O coeficiente de efeito pepita (E\%;) de acordo com Cambardella et al. (1994) mostrou forte dependência espacial para as variáveis microporosidade e densidade do solo $(\mathrm{E} \% \leq 25)$ e moderada para macroporosidade, porosidade total e umidade do solo $(25 \geq \mathrm{E} \% \geq 75)$.

Tabela 3. Modelos e parâmetros estimados dos semivariogramas experimentais

\begin{tabular}{lccccc}
\hline \multicolumn{1}{c}{ Atributo } & Modelo & $\begin{array}{c}\mathbf{C}_{\mathbf{0}} \\
\text { (efeito pepita) }\end{array}$ & $\begin{array}{c}\mathbf{C}_{\mathbf{0}}+\mathbf{C} \\
\text { (patamar) }\end{array}$ & $\begin{array}{c}\mathbf{A} \\
\text { (alcance) }\end{array}$ & E\%* \\
Macroporosidade & $\mathrm{G}$ & 0,000377 & 0,000569 & 124 & 66 \\
Microporosidade & $\mathrm{E}$ & 0,000049 & 0,000251 & 107 & 19 \\
Porosidade Total & $\mathrm{G}$ & 0,000140 & 0,000318 & 32 & 44 \\
Densidade & $\mathrm{E}$ & 0 & 0,005244 & 73 & 0 \\
Umidade & $\mathrm{G}$ & 0,000177 & 0,000247 & 124 & 72 \\
\hline
\end{tabular}

* E\% - Coeficiente de efeito pepita $\left(C_{d} / C_{0}+C\right) \times 100 ; G$ - Gaussiano; $E$ - Exponencial

Conforme Panosso et al. (2008) o alcance (a) é um parâmetro importante no estudo do semivariograma haja vista que representa a distância máxima em que pontos da mesma variável estão correlacionados espacialmente e, sendo assim, pode-se verificar que a dependência dos dados (alcance, "a" - Tabela 3) se deu em um raio que variou de $32 \mathrm{~m}$ (porosidade total) até $124 \mathrm{~m}$ (macroporosidade e umidade do solo), distâncias essas superiores às encontradas por Schaffrath et al. (2008) e Souza et al. (2004).

Observando os mapas gerados após a interpolação dos dados (Figura 1), constata-se que os valores para macroporosidade (Figura 1A) variaram de 0,06 a $0,09 \mathrm{~m}^{3} \mathrm{~m}^{-3}$, sendo que os menores valores de macroporos $\left(0,06 \mathrm{a} 0,07 \mathrm{~m}^{3} \mathrm{~m}^{-3}\right)$ abrangem grande parte do mapa, estendendo-se da parte central à quase totalidade da região sudeste da área. Os maiores valores $(0,08$ a $0,09 \mathrm{~m}^{3} \mathrm{~m}^{-3}$ ) se concentram principalmente no extremo sudoeste do mapa.

A macroporosidade está correlacionada com o crescimento das plantas e seus valores ótimos se encontram entre 0,20 e $0,30 \mathrm{~m}^{3} \mathrm{~m}^{-3}$. Valores superiores a esses restringem a capacidade de retenção de água do solo e valores inferiores a $0,10 \mathrm{~m}^{3} \mathrm{~m}^{-3}$ prejudicam o desenvolvimento das raízes, a infiltração de água e aeração do solo (Kiehl, 1979). Esta variável teve seus valores
A.

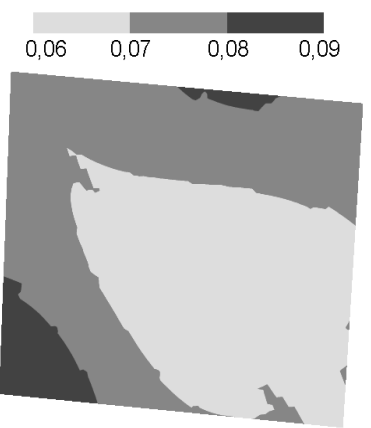

D.

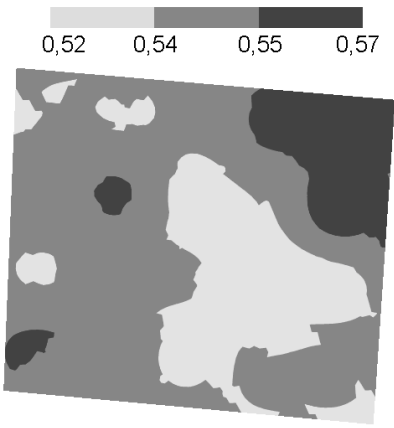<smiles></smiles>

B.

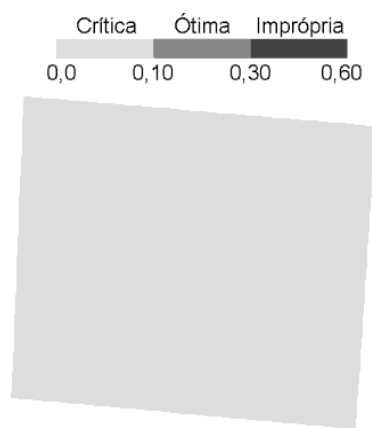

E.
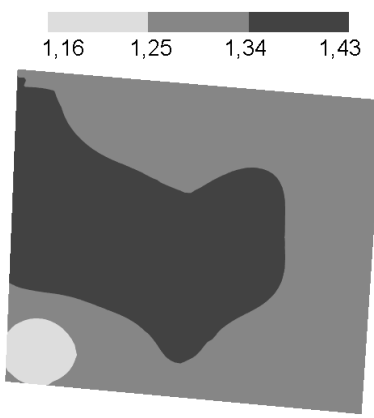

G.

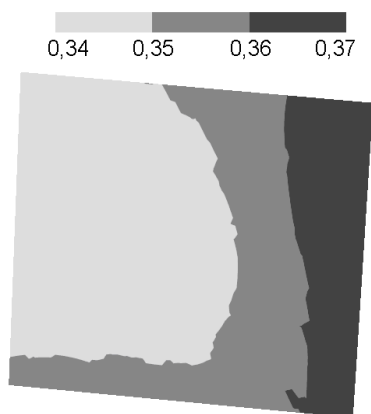

C.

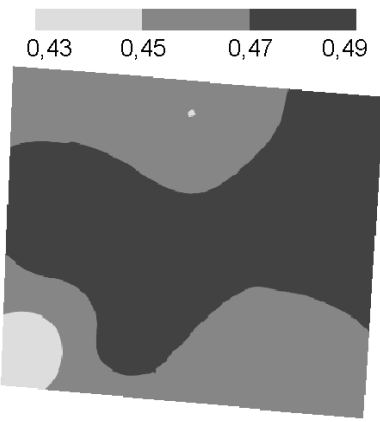

F.
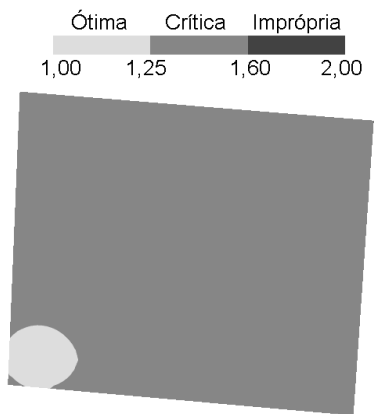

Figura 1. Distribuição espacial da macroporosidade, $\mathrm{m}^{3} \mathrm{~m}^{-3}(\mathrm{~A})$; macroporosidade reclassificada, $\mathrm{m}^{3} \mathrm{~m}^{-3}(\mathrm{~B})$; microporosidade, $\mathrm{m}^{3} \mathrm{~m}^{-3}(\mathrm{C})$; porosidade total, $\mathrm{m}^{3} \mathrm{~m}^{-3}(\mathrm{D})$; densidade, $\mathrm{kg} \mathrm{dm}^{-3}(\mathrm{E})$; densidade reclassificada, $\mathrm{kg} \mathrm{dm}^{-3}(\mathrm{~F})$; umidade do solo, $\mathrm{m}^{3} \mathrm{~m}^{-3}(\mathrm{G})$ 
reclassificados (Figura 1B) segundo os valores apresentados por Kiehl (1979). Utilizou-se o valor máximo igual a $0,60 \mathrm{~m}^{3} \mathrm{~m}^{-3}$ exclusivamente para classificação da legenda. Todos os valores encontrados se mantiveram dentro da mesma faixa de classificação (abaixo de $0,10 \mathrm{~m}^{3} \mathrm{~m}^{-3}$ ), representando uma situação prejudicial ao perfeito desenvolvimento das plantas.

Os valores de microporosidade (Figura $1 \mathrm{C}$ ) variaram de 0,43 a $0,49 \mathrm{~m}^{3} \mathrm{~m}^{-3}$, com os maiores valores $\left(0,47\right.$ a $\left.0,49 \mathrm{~m}^{3} \mathrm{~m}^{-3}\right)$ na maior parte do mapa, estendendo-se desde o sudoeste ao nordeste da área. Os menores valores de microporosidade $(0,43$ a $0,45 \mathrm{~m}^{3} \mathrm{~m}^{-3}$ ) se concentraram quase que exclusivamente em uma pequena região no extremo sudoeste do mapa.

No mapa da porosidade total (Figura 1D) os valores variaram de 0,52 a $0,57 \mathrm{~m}^{3} \mathrm{~m}^{-3}$, predominando valores intermediários de poros totais $\left(0,54\right.$ a $\left.0,55 \mathrm{~m}^{3} \mathrm{~m}^{-3}\right)$ e com menores valores $(0,52 \mathrm{a}$ $\left.0,54 \mathrm{~m}^{3} \mathrm{~m}^{-3}\right)$ na região centro-leste enquanto os maiores valores $\left(0,55\right.$ a $\left.0,57 \mathrm{~m}^{3} \mathrm{~m}^{-3}\right)$ se concentraram sobretudo na região nordeste da área.

Os valores de densidade do solo observados no mapa (Figura 1E) variaram de $1,16 \mathrm{a} 1,43 \mathrm{~kg} \mathrm{dm}^{-3}$, sendo que os valores mais altos $\left(1,34 \mathrm{a} 1,43 \mathrm{~kg} \mathrm{dm}^{-3}\right)$ se encontraram desde o centro até a região noroeste da área. Os menores valores (1,16 a 1,25 $\mathrm{kg} \mathrm{dm}^{-3}$ ) se restringiram a uma pequena região no extremo sudoeste e os valores intermediários de densidade (1,25 a 1,34 $\mathrm{kg} \mathrm{dm}^{-3}$ ) prevaleceram no restante do mapa.

A amplitude média de variação da densidade aparente para solos argilosos foi de 1,00 a $1,25 \mathrm{~kg} \mathrm{dm}^{-3}$, sendo $1,60 \mathrm{~kg} \mathrm{dm}^{-3} \mathrm{o}$ valor limite, acima do qual as raízes não podem penetrar nos solos argilosos (Kiehl, 1979). A densidade do solo (Figura 1E) foi reclassificada (Figura 1F) utilizando-se esses parâmetros e se considerando valores intermediários de 1,25 a $1,60 \mathrm{~kg} \mathrm{dm}^{-3}$ como sendo densidades críticas para as quais o solo deve ser manejado de maneira a evitar aumento da densidade e, em consequência, a compactação. Utilizou-se o valor máximo igual a $2,00 \mathrm{~kg} \mathrm{dm}^{-3}$ exclusivamente para classificação da legenda.

Verificou-se que a maior parte da área se apresenta com valores de densidade considerados críticos, com necessidade de adoção de um manejo adequado. Hamza \& Anderson (2005) sugeriram uma combinação de práticas de manejo para abrandar ou retardar o problema de compactação do solo. Tais práticas incluem o tráfego controlado de máquinas agrícolas, o plantio direto, o uso de rotação de culturas que inclua plantas de raízes profundas, fortes (capazes de penetrar em solos relativamente compactados) e que ofereçam boa cobertura e matéria orgânica para o solo (melhorando não apenas as condições físicas mas também condições químicas e biológicas do solo e contribuindo no controle de plantas invasoras).

A área vinha sendo manejada sob sistema plantio direto há 10 anos, sem um planejamento adequado de rotação de culturas que incluisse plantas de cobertura. Conforme Rosa Filho et al. (2009), no sistema plantio direto, apesar do solo ser submetido a um tráfego menor de máquinas (comparado com o sistema convencional), ele não é revolvido e isto pode provocar adensamento de sua camada superficial. $\mathrm{O}$ adensamento foi verificado pelo aumento da densidade do solo e da microporosidade, da diminuição da porosidade total e, principalmente, da macroporosidade.

A umidade do solo (Figura $1 \mathrm{G}$ ) apresentou valores crescentes do oeste para o leste do mapa, com valores entre 0,34 a $0,35 \mathrm{~m}^{3} \mathrm{~m}^{-3}$ prevalecendo em toda região noroeste e até a região central. Em toda a borda oeste da área os valores foram de 0,36 a $0,37 \mathrm{~m}^{3} \mathrm{~m}^{-3}$.

Nos mapas temáticos correspondentes observou-se comportamento inverso entre a densidade e a macroporosidade; já para a microporosidade e densidade do solo notou-se que a região que possui índices baixos foi a mesma para os dois atributos e regiões com altos índices de macroporosidade possuem baixos índices de microporosidade.

As correlações lineares de Pearson (Tabela 4) e nãoparamétrica de Spearman (Tabela 5) apresentaram tendências similares às observadas nos mapas temáticos. Observou-se fraca correlação linear positiva entre densidade e microporosidade e fraca correlação linear negativa entre macroporosidade e densidade; já entre macroporosidade e microporosidade e entre densidade e umidade do solo, verificou-se forte correlação linear negativa, em discordância com os resultados encontrados por Lopes et al. (2007) para densidade e umidade solo porém concordando com Kiehl (1979), o qual explicou que a diminuição da densidade aparente significa o predomínio de partículas mais finas no solo, com maior capacidade de retenção de água. Também se detectou uma correlação entre macroporosidade e porosidade

Tabela 4. Coeficientes de correlação linear de Pearson para macroporosidade, microporosidade, porosidade total, densidade, umidade do solo

\begin{tabular}{|c|c|c|c|c|c|}
\hline & Microporosidade & Densidade & Macroporosidade & Porosidade Total & $0 \leq|R|<0,2$ (muito fraca) \\
\hline Densidade & $0,385^{\text {ns }}$ & & & & $0,2 \leq|\mathrm{R}|<0,4$ (fraca) \\
\hline Macroporosidade & $-0,670^{*}$ & $-0,389^{\text {ns }}$ & & & $0,4 \leq|\mathrm{R}|<0,6$ (moderada) \\
\hline Porosidade total & $0,046^{\text {ns }}$ & $-0,119^{\text {ns }}$ & $0,668^{*}$ & & $0,6 \leq|\mathrm{R}|<0,8$ (forte) \\
\hline Umidade & $0,407^{n s}$ & $-0,654^{*}$ & $-0,173^{\text {ns }}$ & $0,143^{\text {ns }}$ & $0,8 \leq|R| \leq 1$ ( muito forte) \\
\hline
\end{tabular}

* Significativo pelo teste t ao nível de $5 \%$ de probabilidade

Tabela 5. Coeficientes de correlação não-paramétrica de Spearman para macroporosidade, microporosidade, porosidade total, densidade, umidade do solo

\begin{tabular}{|c|c|c|c|c|c|}
\hline & Microporosidade & Densidade & Macroporosidade & Porosidade Total & $0 \leq|R s|<0,2$ (muito fraca) \\
\hline Densidade & $0,300^{\text {ns }}$ & & & & $0,2 \leq|\mathrm{Rs}|<0,4$ (fraca) \\
\hline Macroporosidade & $-0,428^{\text {ns }}$ & $-0,315^{\text {ns }}$ & & & $0,4 \leq|\mathrm{Rs}|<0,6$ (moderada) \\
\hline Porosidade total & $0,156^{\text {ns }}$ & $-0,119^{\text {ns }}$ & $0,751^{*}$ & & $0,6 \leq \mid$ Rs $\mid<0,8$ (forte) \\
\hline Umidade & $0,331^{\text {ns }}$ & $-0,661^{*}$ & $-0,106^{\text {ns }}$ & $0,071^{\text {ns }}$ & $0,8 \leq|\mathrm{Rs}| \leq 1$ (muito forte) \\
\hline
\end{tabular}

* Significativo pelo teste t a nível de $5 \%$ de probabilidade 
total, resultados que estão de acordo com Schaffrath et al. (2008).

A constatação de diferença entre os coeficientes de correlação linear e o de Spearman, reflete não apenas uma relação não-linear como também a presença de pares de valores extremos, mesmo que este fato não tenha sido observado neste trabalho.

\section{ConclusõEs}

1. A dependência espacial variou entre moderada (macroporosidade, porosidade total e umidade do solo) e forte (microporosidade e densidade do solo).

2. O coeficiente de variação indicou alta variabilidade somente para a variável macroporosidade do solo.

\section{AgradeCimentos}

À Coordenação de Aperfeiçoamento de Pessoal de Nível Superior (CAPES) pela concessão de bolsa de estudo.

\section{LITERATURA CITADA}

Bazzi, C. L.; Souza, E. G.; Uribe-Opazo, M. A.; Santos, D. Uso da validação cruzada na seleção de modelos de semivariogramas. In: Congresso Brasileiro de Engenharia Agrícola, 38, 2009, Petrolina/Juazeiro. Anais... Jaboticabal: SBEA, 2009. CD Rom

Cambardella, C. A.; Moorman, T. B.; Novak, J. M.; Parkin, T. B.; Karlen, D. L.; Turco, R. F.; Konopka, A. E. Field-scale variability of soil properties in Central Iowa soils. Soil Science Society of America Journal, v.58, p.1501-1511, 1994.

Clark, I. Practical geoestatistics. London: Applied Science Publishers Ltda., 1979. 123p.

EMBRAPA - Empresa Brasileira de Pesquisa Agropecuária. Manual de métodos de análise de solo. 2.ed. Rio de Janeiro: CNPS, 1997. 212p.

EMBRAPA - Empresa Brasileira de Pesquisa Agropecuária. Sistema brasileiro de classificação de solos. 2.ed. Rio de Janeiro: CNPS, 2006. 306p.

Faraco, M. A.; Uribe-Opazo, M. A.; Silva, E. A. A.; Johann, J. A.; Borssoi, J. A. Seleção de modelos de variabilidade espacial para elaboração de mapas temáticos de atributos físicos do solo e produtividade da soja. Revista Brasileira de Ciências do Solo, v.32, p.463-476, 2008.

Goedert, W. J.; Schermack, M. J.; Freitas de, F. C. Estado de compactação do solo em áreas cultivadas no sistema de plantio direto. Pesquisa Agropecuária Brasileira, v.37, p.223227, 2002.

Gomes, F. P.; Garcia, C. H. Estatística aplicada a experimentos agronômicos e florestais, Piracicaba: FEALQ, 2002. 305p.

Gonçalves, A. C. A.; Folegatti, M. V.; Mata, J. D. V. da. Análises exploratória e geoestatística da variabilidade de propriedades físicas de um Argissolo Vermelho. Acta Scientiarum Agronomy, v.23, p.1149-1157, 2001.
Hamza, M. A.; Anderson, W. K. Soil compaction in cropping systems: A review of the nature, causes and possible solutions. Soil and Tillage Research, v.82, p.121-145, 2005.

IAPAR - Instituto Agronômico do Paraná. Características geográficas do município de Cafelândia.: <http:// www.cafelandia.pr.gov.br/cafelandia>. 4 Mar. 2009.

Jones, T. A. Skewness and kurtosis as criteria of normality in observed frequency distributions. Journal of Sedimentary Petrology, v.39, p.1622-1627, 1969.

Kiehl, E. J. Manual de edafologia: Relações solo-planta. 1.ed. São Paulo: Agronômica Ceres, 1979. 264p.

Kiliç, K.; Özgöz, E.: Akba, F. Assessment of spatial variability in penetration resistance as related to some soil physical properties of two ûuvents in Turkey. Soil and Tillage Research, v.76, p.1-11, 2004.

Lopes, R. A. P.; Nóbrega, L. H. P.; Uribe-Opazo, M. A.; Prior, M.; Pereira, J. O. Propriedades físicas de Latossolo Vermelho distroférrico típico sob sistema de manejo na sucessão sojamilho no período de três anos. Acta Scientiarum Agronomy, v.29, p.721-727, 2007.

Megda, M. M.; Carvalho, M. P.; Vieira, M. X.; Andreotti, M.; Pereira, E. C. Correlação linear e espacial entre a produtividade de feijão e a porosidade de um Latossolo Vermelho de Selvíria (MS). Revista Brasileira de Ciência do Solo, v.32, p.781-788, 2008.

Panosso, A. R.; Pereira, G. T.; Marques Júnior, J. ; La Scala Júnior, N. Variabilidade espacial da emissão de $\mathrm{CO}_{2}$ em Latossolos sob cultivo de cana-de-açúcar em diferentes sistemas de manejo. Engenharia Agrícola, v.28, p.227-236, 2008.

Ramirez-Lopez, L.; Reina-Sanchez, A.; Camacho-Tamayo, J. H. Variabilidad espacial de atributos físicos de un Typic Haplustox de los Llanos Orientales de Colômbia. Engenharia Agrícola, v.28, p.55-63, 2008.

Rosa Filho, G.; Carvalho, M. P.; Andreotti, M.; Montanari, R.; Binotti, F. F. S.; Gioia, M. T. Variabilidade da produtividade da soja em função de atributos físicos de um Latossolo Vermelho distroférrico sob plantio direto. Revista Brasileira de Ciência do Solo, v.33, p.283-293, 2009.

Schaffrath, V. R.; Tormena, C. A.; Gonçalves, A. C. A.; Fidalski, J. Variabilidade e correlação espacial de propriedades físicas de solo sob plantio direto e preparo convencional. Revista Brasileira de Ciência do Solo, v.32, p.1369-1377, 2008.

Souza, Z. M.; Marques, J. J.; Cooper, M.; Pereira G. T. Micromorfologia do solo e sua relação com atributos físicos e hídricos. Pesquisa Agropecuária Brasileira, v.41, p.487492, 2006.

Souza, Z. M.; Marques, J. J.; Pereira G. T.; Bento, M. J. C. Variabilidade espacial de atributos físicos de um Latossolo Vermelho eutroférrico. Revista Brasileira de Engenharia Agrícola e Ambiental, v.8, p.51-58, 2004.

Thimóteo, C. M. S.; Beninni, E. R. Y.; Murata, I. M.; Tavares, J. F. Alterações da porosidade e da densidade de um Latossolo Vermelho Distrófico em dois sistemas de manejo de solo. Acta Scientiarum Agronomy, v.23, p.1299-1303, 2001.

Vieira, M. L.; Klein, V. A. Propriedades físico-hídricas de um Latossolo Vermelho submetido a diferentes sistemas de manejo. Revista Brasileira de Ciência do Solo, v.31, p.12711280, 2007.

Zou, K. H.; Tuncali, K.; Silverman, S. G. Correlation and simple linear regression. Radiology, v.227, p.617-628, 2003. 